\title{
Detectability of dirty dust grains in brown dwarf atmospheres
}

\author{
Ch. Helling ${ }^{1}$, W.-F. Thi ${ }^{1,3}$, P. Woitke ${ }^{2}$, and M. Fridlund ${ }^{1}$ \\ ${ }^{1}$ Research and Scientific Support Department, ESTEC/ESA, PO Box 299, 2200 AG Noordwijk, The Netherlands \\ e-mail: chelling@esa.int \\ 2 Sterrewacht Leiden, PO Box 9513, 2300 RA Leiden, The Netherlands \\ 3 Institute for Astronomy, University of Edinburgh, Blackford Hill, Edinburgh EH9 3HJ, UK
}

Received 1 February 2006 / Accepted 11 March 2006

\section{ABSTRACT}

Context. Dust clouds influence the atmospheric structure of brown dwarfs, and they affect the heat transfer and change the gas-phase chemistry. However, the physics of their formation and evolution is not well understood. The dust composition can be predicted from thermodynamical equilibrium or time-dependent chemistry that takes into account seed particle formation, grain growth, evaporation, and drift.

Aims. In this Letter, we predict dust signatures and propose a potential observational test of the physics of dust formation in brown dwarf atmospheres based on the spectral features of the different solid components predicted by dust formation theory.

Methods. A momentum method for the formation of dirty dust grains (nucleation, growth, evaporation, and drift) is applied to a static brown dwarf atmosphere structure to compute the dust grain properties, and in particular, the heterogeneous grain composition and the grain size. The effective medium and Mie theories are used to compute the extinction of these spherical grains.

Results. Dust formation results in grains whose composition differs from that of grains formed at equilibrium. Our kinetic model predicts that amorphous $\mathrm{SiO}_{2}[\mathrm{~s}]$ (silica) is one of the most abundant solid components, followed by amorphous $\mathrm{Mg}_{2} \mathrm{SiO}_{4}[\mathrm{~s}]$ and $\mathrm{MgSiO}_{3}[\mathrm{~s}]$, while $\mathrm{SiO}_{2}[\mathrm{~s}]$ is absent in equilibrium models because it is a metastable solid. Solid amorphous $\mathrm{SiO}_{2}$ [s] possesses a strong broad absorption feature centered at $8.7 \mu \mathrm{m}$, while amorphous $\mathrm{Mg}_{2} \mathrm{SiO}_{4}[\mathrm{~s}] / \mathrm{MgSiO}_{3}[\mathrm{~s}]$ absorbs at $9.7 \mu \mathrm{m}$ in addition to other absorption features at longer wavelengths. Those features at $\lambda<15 \mu \mathrm{m}$ are detectable in absorption if the grains are small (radius $<0.2 \mu \mathrm{m}$ ) in the upper atmosphere, as proposed by our model. Conclusions. We suggest that the detection of a feature at $8.7 \mu \mathrm{m}$ in deep infrared spectra could provide evidence for non-equilibrium dust formation that yields grains composed of metastable solids in brown dwarf atmospheres. This feature will shift towards $10 \mu \mathrm{m}$ and broaden if silicates (e.g. fosterite) are much more abundant.

Key words. astrochemistry - methods: numerical - stars: atmospheres - stars: low-mass, brown dwarfs - infrared: stars

\section{Introduction}

Atmospheric models are essential to the interpretation of complex brown dwarf's spectra, which are dominated by strong molecular absorption lines. The physics and chemistry of substellar objects (brown dwarfs and giant planets) are more complicated than anticipated because of their nonequilibrium processes (dust formation and convective mixing) and their non-linear feedbacks on radiative transfer. Consequently, parametrisations of processes like dust formation and convection were applied in atmospheric models until now. Current model atmospheres provide extensive solutions for the radiative transfer problem of the gas phase in hydrostatic equilibrium (Marley et al. 1996; Tsuji et al. 1996; Burrows et al. 1997; Allard et al. 2001). However, the presence of dust is currently only inferred from classical models, where the dust composition is derived from gas/solid phase equilibrium considerations and time-scale arguments, and their comparison to observed spectra (Lunine et al. 1989; Tsuji et al. 1996; Tsuji 2002; Ackerman \& Marley 2001; Allard et al. 2001; Cooper et al. 2003). Such equilibrium chemistry results in grains with a homogeneous composition.

Considering the non-equilibrium character of phase transitions (supersaturation $\gg 1$ ), Woitke \& Helling $(2003,2004)$ and Helling \& Woitke (2006) proposed a theoretical approach to consistently model the formation of dust grains by seed formation, growth, evaporation, and drift (gravitational settling). In contrast to the phase-equilibrium calculation, Woitke $\&$ Helling argue that grains in the oxygen-rich environment of a brown dwarf are heterogeneous in chemical composition and in size. Moreover, precipitating into the denser inner atmospheres, these particles can reach sizes of several $100 \mu \mathrm{m}$. In Helling \& Woitke (2006), the inferred dust composition differs markedly from that predicted by equilibrium chemistry. These differences manifest themselves in the intrinsic absorption signatures of the solids in the mid-infrared range. 
This Letter suggests a potential observational test for the presence of dust. The dust may be present in form of cloud-like structures. We will focus on the main characteristics of the theory, by which the predictions in terms of dust spectral features can be tested with current and future instruments. In Sect. 2, we report on recent progress in modeling the formation of chemically non-homogeneous cloud layers made of dirty (i.e. made up of a variety of compounds) grains in brown dwarf atmospheres. A detailed Mie theory treatment combined with effective medium theory allows us to suggest possible spectral features of such a dust layer for both approaches. The result of the modeling is described and discussed in Sect. 3

\section{Approach}

We model nucleation (seed formation), heterogeneous growth, evaporation, and drift (gravitational settling) of dirty dust particles in a quasi-static atmosphere by using the moment method (Helling \& Woitke 2006). We consider the formation of compact spherical grains in an oxygen-rich gas by the initial nucleation of $\mathrm{TiO}_{2}$ seed particles, followed by the growth of a dirty mantle made of various solids. We consider amorphous $\mathrm{TiO}_{2}[\mathrm{~s}], \mathrm{SiO}_{2}[\mathrm{~s}], \mathrm{MgO}$ [s], $\mathrm{MgSiO}_{3}[\mathrm{~s}], \mathrm{Mg}_{2} \mathrm{SiO}_{4}$ [s], $\mathrm{Al}_{2} \mathrm{O}_{3}$ [s], and metallic iron $\mathrm{Fe}[\mathrm{s}]$, assuming that silica and silicates have approximately the same sticking probability. The moment and elemental conservation equations for $\mathrm{Ti}, \mathrm{Si}, \mathrm{Mg}, \mathrm{Fe}, \mathrm{Al}$, and $\mathrm{O}$ are evaluated using $\left(T, \rho, v_{\text {conv }}\right)$ of a prescribed static model atmosphere structure (Allard et al. ${ }^{1}$ ) without further iteration on the temperature profile. The model calculates the amount of condensates, the mean grain size $\langle a\rangle$, and the volume fractions $V_{\mathrm{s}}$ of each material as a function of height $z$ in the brown dwarf atmosphere for given model structures. At each depth in the atmosphere, the volume fractions are used to compute mean dust optical constants using the Maxwell-Garnett effective medium theory (Bohren \& Huffman 1983), which is a valid method because the grains generally have one main volume component. The main dust component is the matrix in which the spherical inclusions made of the minor components are embedded. Dust extinction coefficients are subsequently computed using a Mie theory code for compact spherical grains. The different components are assumed to be in an amorphous state, consistent with laboratory experiments of heterogeneous dust formation (e.g., Rietmeijer et al. 1999). The optical constants for the amorphous solids are obtained from laboratory measurements $\left(\mathrm{TiO}_{2}\right.$ [s]: Ribarsky $1985 ; \mathrm{SiO}_{[\mathrm{s}]}$ : Henning \& Mutschke 1997; MgO: Hofmeister et al. 2003; $\mathrm{MgSiO}_{3}[\mathrm{~s}] / \mathrm{Mg}_{2} \mathrm{SiO}_{4}$ [s]: Jäger et al. 2003; $\mathrm{Al}_{2} \mathrm{O}_{3}$ [s]: Begemann et al. 1997; and $\mathrm{Fe}[\mathrm{s}]$ : Ordal et al. 1985). Absorption cross sections and optical depth are then derived using the dust density profiles and assuming a grain size distribution $f(a, z)=\langle a(z)\rangle \delta(a-\langle a(z)\rangle)$. For comparison, similar computations were performed assuming that no $\mathrm{SiO}_{2}[\mathrm{~s}]$ can form by setting the formation rate to zero. We also estimated the possible absorption depth in observed spectra. The contrast between the depth of the features and the continuum is an important parameter for predicting the detectability of the dust features.

\footnotetext{
${ }^{1}$ Adopted from ftp.ens-lyon.fr/pub/users/CRAL/fallard/
}

\section{Results}

We first show the grain size and dust composition profile of an AMES (cond) model with $T_{\text {eff }}=1500 \mathrm{~K}, \log g=5.0\left(^{1}\right)$, and a solar element composition. This can be considered a typical late L-dwarf atmosphere model. The outputs are then used to compute the dust extinction coefficient in the mid-infrared.

\subsection{Global grain size distribution and material composition}

The results depicted in Fig. 1 show the same global dust-cloud structure as presented in Woitke \& Helling (2004). The formation of the cloud is governed by the hierarchical dominance of nucleation (uppermost layers), growth and drift (intermediate layers), and evaporation and drift (deepest layers). The uppermost cloud layers are predominantly filled with small grains of a mean size of $10^{-2} \mu \mathrm{m}$, which grow on their way into the atmosphere to 200 ...300 $\mu \mathrm{m}$ (1.h.s. Fig. 1). Eventually, they enter even hotter atmospheric layers where they are no longer thermally stable. Hence, the grains shrink in size and finally dissolve into the surrounding hot, convective gas. This picture reflects the stationary character of the grain component forming the brown dwarf's dust cloud, where dusty material constantly falls inward and fresh, dust-free material is mixed upward.

The r.h.s. of Fig. 1 demonstrates the chemical composition of the dust grains. The grains are not of a single, homogeneous material composition, and their material composition changes on their descent into the atmosphere. Since gas and dust thermalise faster than the dust growth/evaporation processes take place (Woitke \& Helling 2003), the chemical composition is determined by the local temperature and the reaction kinetics. Therefore, the upper atmosphere is populated by small, silicaand silicate-like grains until these materials become thermally unstable. Therefore, in the lower and hotter part of the atmosphere, big grains appear that are merely made of iron and some impurities of $\mathrm{Al}_{2} \mathrm{O}_{3}[\mathrm{~s}]$ and $\mathrm{TiO}_{2}[\mathrm{~s}]$. The main dust component in the upper part of the dust layer is amorphous $\mathrm{SiO}_{2}$ [s], followed by $\mathrm{Mg}_{2} \mathrm{SiO}_{4}[\mathrm{~s}] / \mathrm{MgSiO}_{3}[\mathrm{~s}]$ (see Table 1 ). This prediction contrasts with those by equilibrium dust formation models, where metastable species such as $\mathrm{SiO}_{2}[\mathrm{~s}]$ cannot exist (e.g. Lodders \& Fegley 2005, in press).

\subsection{Spectral dust cloud features}

Figure 2 depicts the resulting spectral features from $6 \mu \mathrm{m} \ldots$ $15 \mu \mathrm{m}$ for a brown dwarf dust-cloud layer with the mean grain size and chemical dust material composition shown in Fig. 1.

For grain composition predicted by heterogeneous dust formation, the only dust features with an appreciable contrast are those of $\mathrm{SiO}_{2}$ [s] centered at $8.7 \mu \mathrm{m}$ and of $\mathrm{Mg}_{2} \mathrm{SiO}_{4}[\mathrm{~s}] / \mathrm{MgSiO}_{3}[\mathrm{~s}]$ at $9.7 \mu \mathrm{m}$, with weaker absorption features around $20 \mu \mathrm{m}$ and $32 \mu \mathrm{m}$ (not shown). The features are broad $(\sim 1-1.5 \mu \mathrm{m})$ and lack substructures because the grains are amorphous. The abundance of metallic iron is high $(\sim 15 \%)$, but metals absorb photons continuously and do not show spectral features. The abundance of $\mathrm{Al}_{2} \mathrm{O}_{3}[\mathrm{~s}]$ and $\mathrm{TiO}_{2}[\mathrm{~s}]$ in grains is too small to significantly affect the overall 

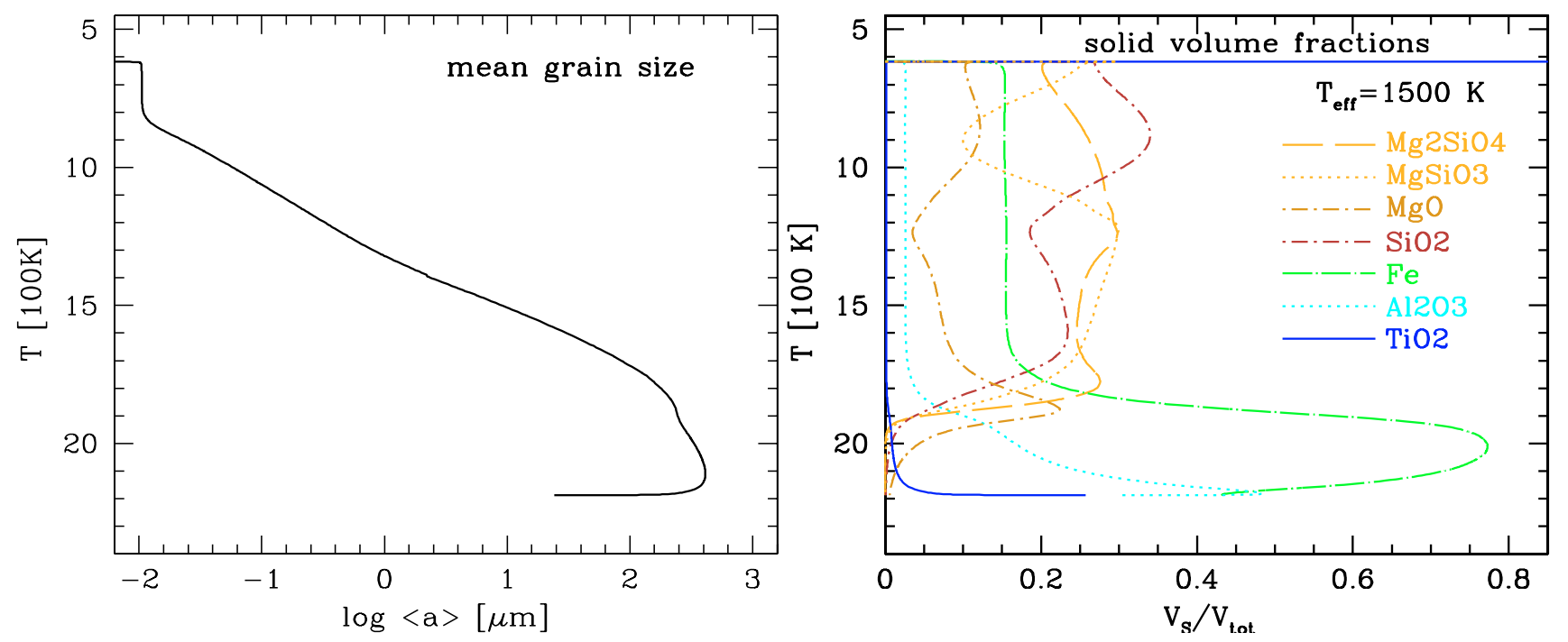

Fig. 1. Left: mean grain size stratification for a plan-parallel AMES (cond) late L-type brown dwarf model atmosphere of $T_{\text {eff }}=1500 \mathrm{~K}$, $\log g=5.0$ and of original solar abundance. Right: chemical material composition of the grains in volume fractions of the solids for the same model $\left(V_{\mathrm{tot}}^{\text {dust }}=\sum_{\mathrm{s}} V_{\mathrm{s}}, \mathrm{s}-\right.$ contributing solid materials). Note the upside-down scale of the local temperature $T[100 \mathrm{~K}]$.

extinction coefficient. In models where $\mathrm{SiO}_{2}[\mathrm{~s}]$ is disregarded (dashed line, 1.h.s. Fig. 2), the dust extinction is dominated by $\mathrm{Mg}_{2} \mathrm{SiO}_{4}[\mathrm{~s}] / \mathrm{MgSiO}_{3}[\mathrm{~s}]$ at $9.7 \mu \mathrm{m}$. The mean grain radius remains smaller than $0.2 \mu \mathrm{m}$ in the $\tau_{\text {dust }}<1$ region in the model. Therefore, the grain absorption features remain. In contrast, if the grains were as large as $10 \mu \mathrm{m}$, the resonance features would disappear (Min et al. 2004). The region where $\tau_{\text {dust }}<1$, in the wavelength range 5-25 $\mu \mathrm{m}$, has pressure below $10^{-2}$ bar in the model atmosphere considered.

In phase-equilibrium, the condensates are formed locally and retain the energetically most-favorable composition at the temperature and pressure of the given depth in the atmosphere (Lodders \& Fegley 2005, in press). Further condensation is not possible because the grains are kept in relatively thin, discrete cloud layers and, hence, cannot move into regions with still favorable growth conditions. Individual cloud layers are composed of different solid species, depending on the temperature of the layer. Above the silicate clouds, the gas phase is strongly depleted, and thus the absence of gas-phase species in $\mathrm{L}$ and $\mathrm{T}$ dwarf atmospheres has been used as evidence of the presence of clouds. The prediction of equilibrium theory is the absence of solid $\mathrm{SiO}_{2}$; hence equilibrium dust is predominately composed of silicates. We modeled the absence of $\mathrm{SiO}_{2}[\mathrm{~s}]$, and the spectral result is shown in Fig. 2 (1.h.s.). Besides moving the peak of the absorption to $9.7 \mu \mathrm{m}$, the absence of amorphous $\mathrm{SiO}_{2[\mathrm{~s}]}$ substantially decreases the absorption contrast.

\subsection{Discussion of detectability}

We now discuss the detectability of infrared dust features in brown dwarf atmospheres. From Fig. 2 (r.h.s.), we can see that the absorption over continuum contrast varies from $10 \ldots 20 \%$, with the maximum obtained for cold brown dwarfs, if $\mathrm{SiO}_{2}[\mathrm{~s}]$ is present. This contrast is achieved because the grains are small $(<0.2 \mu \mathrm{m})$. In the absence of $\mathrm{SiO}_{2}[\mathrm{~s}]$, $\mathrm{Mg}_{2} \mathrm{SiO}_{4}[\mathrm{~s}] / \mathrm{MgSiO}_{3}[\mathrm{~s}]$ will weakly absorb at $9.7 \mu \mathrm{m}$. In the
Table 1. Mean size and material composition of dust grains.

\begin{tabular}{|c|c|c|}
\hline $\begin{array}{l}\text { Local } \\
\text { temperature } \\
T[\mathrm{~K}]\end{array}$ & $\begin{array}{l}\text { Mean grain } \\
\text { size } \\
\langle a\rangle[\mu \mathrm{m}]\end{array}$ & $\begin{array}{l}\text { Material } \\
\text { composition } \\
\text { [volume frac.] }\end{array}$ \\
\hline$\lesssim 1000$ & $10^{-2} \ldots 10^{-1}$ & $\begin{array}{l}25-35 \% \mathrm{SiO}_{2}[\mathrm{~s}] \\
20-25 \% \mathrm{Mg}_{2} \mathrm{SiO}_{4}[\mathrm{~s}] \\
10-25 \% \mathrm{MgSiO}_{3}[\mathrm{~s}] \\
15 \% \mathrm{Fe}[\mathrm{s}], 10 \% \mathrm{MgO}[\mathrm{s}]\end{array}$ \\
\hline $1000 \ldots 1700$ & $10^{-1} \ldots 30$ & $\begin{array}{l}25-30 \% \mathrm{Mg}_{2} \mathrm{SiO}_{4}[\mathrm{~s}] \\
20-30 \% \mathrm{MgSiO}_{3}[\mathrm{~s}] \& \mathrm{SiO}_{2}[\mathrm{~s}] \\
15 \% \mathrm{Fe}[\mathrm{s}], 10 \% \mathrm{MgO}[\mathrm{s}]\end{array}$ \\
\hline $1700 \ldots 2000$ & $30 \ldots 200$ & strongly changing composition \\
\hline $2000 \ldots 2300$ & $200 \ldots 300$ & $80 \% \mathrm{Fe}[\mathrm{s}], \sim 18 \% \mathrm{Al}_{2} \mathrm{O}_{3}$ \\
\hline
\end{tabular}

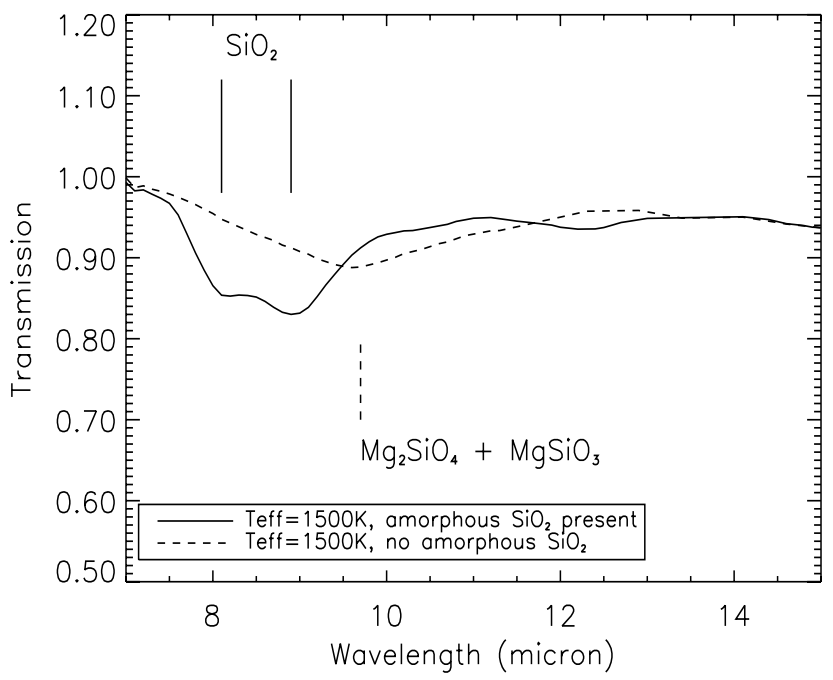

Fig. 2. Absorption transmission spectrum $\left(=F_{v} / F_{\text {cont }}\right.$, with $F_{\text {cont }}$ as the local continuum flux with respect to the feature) for the model in Fig. 1 with (solid line) and without (dashed line) formation of $\mathrm{SiO}_{2}[\mathrm{~s}]$. The absorption feature of $\mathrm{SiO}_{2}[\mathrm{~s}]$ is centered at $\sim 8.7 \mu \mathrm{m}$. 
case of large grains, no feature can be detected and it is not possible to determine the composition of these grains. The presence or absence of $\mathrm{SiO}_{2}$ [s] can be tested observationally. Recently, Cushing et al. (2006) presented Spitzer IRS ${ }^{2}$ results showing L-dwarf spectra with a $1-1.5 \mu \mathrm{m}$ broad absorption centered at $\lambda \approx 9 \mu \mathrm{m}$. However, we have treated an idealized situation where dust grains are the main sources of opacity. In reality, the feature will appear on top of gas absorption lines. The gas phase absorption proceeds by lines whose width is determined by the pressure at the height of the absorption layer. Typical molecular band widths are $\sim 0.1 \mu \mathrm{m}$.

Another limitation of our treatment is the assumption of spherical grains, which may introduce spurious effects during the opacity calculation. Grains are most likely non-spherical and their absorption coefficients should be computed with, for example, the hollow sphere model (Min et al. 2005), which successfully models asphericity by applying a distribution of hollow spheres. However, the use of hollow spheres would only introduce minor changes in the shape of the absorption feature and thus would not significantly change the wavelengthposition of the dust features and hence the conclusion of this paper. Finally, the heterogeneous formation model does not compute the exact grain size distribution, but only its moments from which the mean size can be computed. Future work is needed to calculate better analytical representations of the sizedistribution function from the dust moments.

\section{Conclusions}

We have studied the dust spectral signatures predicted by the Helling \& Woitke (2006) models that make specific observational predictions in terms of grain composition and size at different altitudes. Heterogeneous dust formation in brown dwarf atmospheres results in grains composed mainly of amorphous $\mathrm{SiO}_{2}[\mathrm{~s}]$ and amorphous $\mathrm{Mg}_{2} \mathrm{SiO}_{4}[\mathrm{~s}] / \mathrm{MgSiO}_{3}[\mathrm{~s}]$, with impurities of $\mathrm{Fe}$ and $\mathrm{Al}$ oxides, $\mathrm{TiO}_{2}$ and $\mathrm{MgO}$. The dust forms one continuous layer in which the grains gradually change composition and size. The grains are sufficiently small in the upper atmosphere to produce solid resonance features in the infrared, a conclusion also reached by Cushing et al. (2006). In contrast, the grain composition derived from chemical equilibrium models is dominated by $\mathrm{Mg}_{2} \mathrm{SiO}_{4}$ [s] with the noticeable absence of amorphous $\mathrm{SiO}_{2}[\mathrm{~s}]$, which is a metastable species. The difference in dust composition allows us to make predictions about dust spectral features in the infrared for the two types of grains. Amorphous $\mathrm{SiO}_{2}$ [s] moves the peak of the absorption to
$8.7 \mu \mathrm{m}$, while a nearly pure $\mathrm{Mg}_{2} \mathrm{SiO}_{4}[\mathrm{~s}] / \mathrm{MgSiO}_{3}[\mathrm{~s}]$ absorbs at $9.7 \mu \mathrm{m}$ with a much smaller contrast. Therefore, infrared features of dust grains could be used to directly detect the presence of dust in brown dwarf atmospheres. The detection of dust absorption features supports the prediction that grains are small in size $(<0.5 \mu \mathrm{m})$ and present in the $\tau_{\text {dust }}<1$ upper atmosphere.

Acknowledgements. We thank the anonymous referee for his suggestions. We thank A. Heras for comments on the manuscript, F. Allard and P. Hauschildt for making their model results freely available. Ch.H. and W.F.T. acknowledge ESA internal fellowships at ESTEC, PW the NWO Computational Physics program, grant 614.031.017, in the ASTROHYDRO3D initiative. The computer support by the ESTEC RSSD computer team is highly appreciated.

\section{References}

Ackerman, A. S., \& Marley, M. S. 2001, ApJ, 556, 872

Allard, F., Hauschildt, P. H., Alexander, D. R., et al. 2001, ApJ, 556, 357

Begemann, B., Dorschner, J., Henning, T., et al. 1997, ApJ, 476, 199

Bohren, C. F., \& Huffman, D. R. 1983, Absorption and scattering of light by small particles (New York: Wiley)

Burrows, A., Marley, M., Hubbard, W. B., et al. 1997, ApJ, 491, 856

Cooper, C. S., Sudarsky, D., Milsom, J. A., et al. 2003, ApJ, 586, 1320

Cushing, M. C., Roellig, T. L., Marley, M. S., et al. 2006, ApJ, submitted

Helling, Ch., \& Woitke, P. 2006, A\&A, submitted

Henning, T., \& Mutschke, H. 1997, A\&A, 327, 743

Hofmeister, A. M., Keppel, E., \& Speck, A. K. 2003, MNRAS, 345, 16

Jäger, C., Dorschner, J., Mutschke, H., Posch, T., \& Henning, T. 2003, A\&A, 408, 193

Lodders, K., \& Fegley, B. 2005, in press, Review for Astrophysics Update

Lunine, J. I., Hubbard, W. B., Burrows, A., Wang, Y.-P., \& Garlow, K. 1989, ApJ, 338, 314

Marley, M. S., Saumon, D., Guillot, T., et al. 1996, Science, 272, 1919 Min, M., Dominik, C., \& Waters, L. B. F. M. 2004, A\&A, 413, L35

Min, M., Hovenier, J. W., \& de Koter, A. 2005, A\&A, 432, 909

Ordal, M. A., Bell, R. J., Alexander, R. W., Long, L. L., \& Querry, M. R. 1985, Appl. Opt., 24, 4493

Ribarsky, M. W. 1985, in Handbook of Optical Constants, ed. E. D. Palik (Orlando: Academic Press), 795

Rietmeijer, F. J. M., Nuth, J. A., \& Karner, J. M. 1999, ApJ, 527, 395

Tsuji, T. 2002, ApJ, 575, 264

Tsuji, T., Ohnaka, K., Aoki, W., \& Nakajima, T. 1996, A\&A, 308, L29

Woitke, P., \& Helling, Ch. 2003, A\&A, 399, 297

Woitke, P., \& Helling, Ch. 2004, A\&A, 414, 335 